\title{
Edge-Based Structural Features for Content-Based Image Retrieval
}

\author{
Xiang Sean Zhou, Thomas S. Huang \\ Beckman Institute for Advanced Science and Technology \\ University of Illinois at Urbana Champaign, Urbana, IL 61801, USA \\ \{xzhou2,huang\}@ifp.uiuc.edu
}

\begin{abstract}
This paper proposes structural features for content-based image retrieval (CBIR), especially edge/structure features extracted from edge maps. The feature vector is computed through a "Water-Filling Algorithm" applied on the edge map of the original image. The purpose of this algorithm is to efficiently extract information embedded in the edges. The new features are more generally applicable than texture or shape features. Experiments show that the new features can catch salient edge/structure information and improve the retrieval performance.
\end{abstract}

Keyword: structural feature; water-filling algorithm; Content based image retrieval; relevance feedback

\section{Introduction}

Content-based image retrieval (CBIR) is an active yet challenging research area. The performance of a CBIR system is inherently constrained by the features adopted to represent the images in the database. Color, texture, and shape are the most frequently referred "visual contents" (Flickner et al., 1995). One of the main difficulties in such systems has to do with the fact that the aforementioned "visual contents", or low-level features, though extractable by computers, often cannot readily represent the high-level concepts in the user's mind during the retrieval process. Therefore the research directions include but not limited to, in one direction, incorporating machine learning and intelligence into the system-The learning can be on-line (e.g., user-in-theloop through relevance feedback) or off-line (e.g. constructing face detector; car detector (Ratan et al., 1999); building detector (Iqbal and Aggarwal, 1999); etc.); and in another direction, exploring low-level features beyond color, texture and shape that can improve the performance of the retrieval system. It is the purpose of this paper to propose structural feature for CBIR system.

If we regard information embedded in a digital image as chrominance information combined with illuminance information, then color feature captures the chrominance information; and both texture and shape represent the illuminance information. Texture features (e.g. cooccurrence features (Haralick et al., 1973) and Wavelet based features (Laine and Fan, 1993)) and shape features (e.g. Fourier descriptors (Zahn and Roskies, 1972) and moment invariants $(\mathrm{Hu}, 1962)$ ) have been applied extensively in image retrieval systems. However, texture features are effective only for uniform texture images or regions, and extraction of shape features require image segmentation and are only effective for images with simple and clean object contours.

Real-world images (e.g., natural scenes) are however with noise, complex backgrounds, or object occlusions, etc. The texture and shape features are therefore far from adequate and are limited to deal with only a small fraction of the real-world images. We therefore propose a new feature set called structural features of which one can think as features in-between texture and shape. Texture captures spatial distribution of illuminance variations and typically pays attention only to "repeating patterns". Whereas shape represents a specific edge feature that is related to object contour. Neither of them pays attention to information represented in non-repeating illuminance patterns in general, which is the category we want to define the structural features to represent. In this sense, we can classify some of the features adopted in the literature as structural features: edge density; edge direction; cotermination ("L" or "U" junctions) and parallel lines (Iqbal and Aggarwal, 1999); spatial/layout relations (Flickner et al., 1995); etc. In this paper we identify a subset of structural features, namely edge/structural features, and provide an algorithm for fast and efficient extraction. Examples of edge/structural features include but not limited to: edge length, edge complexity, loop in the edges, etc., and their statistics 
throughout the image. Even though it is possible for one to argue that there is no clear conceptual boundary between structure and texture or shape features, structural features clearly do not fall into either of the two categories.

In this paper we limit our emphasis on the extraction of structural features from edge maps using our proposed water-filling algorithm. The rest of the paper is organized as follows. In Section 2 background and motivation are described. In Section 3 we introduce with examples the water-filling algorithm. Feature extraction based on the new method is presented in Section 4. Section 5 gives the experimental results over real-world images. Conclusions are drawn in Section 6.

\section{Background and Motivation}

Edge map is usually used for extracting object shape. Shape extraction requires edge linking as the reprocessing step. Without edge linking, "shape" is out of the question; and features such as "edge length" or "edge smoothness" are also hardly meaningful. However, edge linking from an edge map is not trivial at all if we realize that the edge set can be seen as general graphs. A heuristic graph search is not globally optimal thus runs the risk of losing vital information (Jain, 1989). Whereas a globally optimal method such as dynamic programming (DP) or graph theoretic technique may be computationally too expensive. This is the reason that shape is usually difficult to be implemented into a general purpose CBIR system for real world images. Another problem associated with edge map is that edge detectors are usually not ideal in that the edge map does not retain some important information, so that by looking only at an edge map, sometimes even human can not tell what is in the image. So people often hesitate to use edge maps directly thus have ignored the information embedded in them. Because of these reasons only a very limited number of edge/structural features have been explored for CBIR systems, most of them are not used as a general feature but just for specific queries like detecting "city", "buildings", etc.

To illustrate our motivation, consider the edge maps in Fig. 0. In all the three edge maps shape cannot be easily extracted. A human subject can tell the top two as the Lena image and a building image. However one cannot tell the content of the third image based solely on the edge map. What can machines do? Nothing much if "understanding of the content" (i.e., automatic segmentation and recognition of the object) is the goal. But if the goal is image retrieval and matching only, i.e., finding a representation which gives similar "numbers" whenever two images have similar content, and vice versa, the issue becomes "how to effectively and efficiently represent information embedded in the edge map?" Of course based on pure global, low-level representation without any segmentation or contour representation, it is difficult to obtain invariant features for high-level concepts embedded in the image, say "fox', or "flowers". But our intuition is that as long as the edge detector is consistent for all images in the database, one should be able to find similarities in edge maps of images with similar structure.
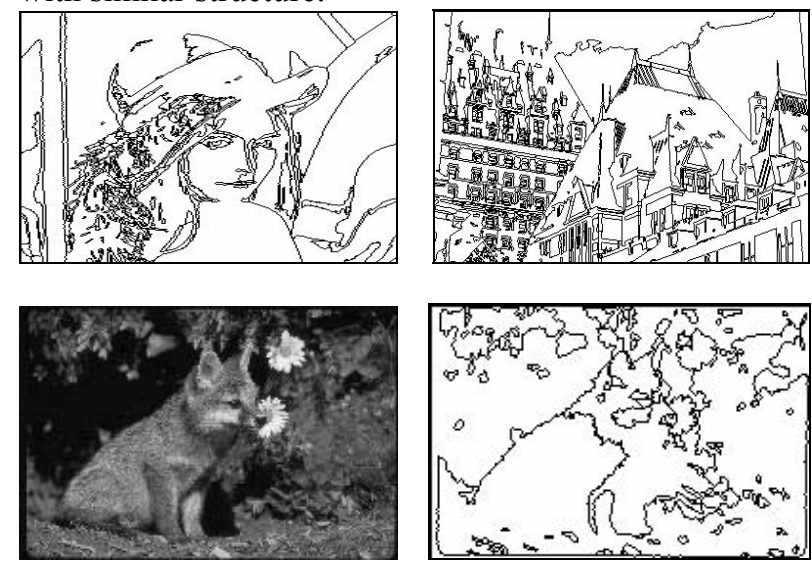

Fig. 0 Examples of edge maps

\section{The Water-Filling Algorithm}

To address the issue raised in Section 2, we propose an algorithm to extract features from the edge map directly without edge linking or shape representation. The idea is to look for measures for the edge length and edge structure and complexity by a very efficient graph traverse algorithm.

In a binary edge map, two edge points are 4-connected (or 4-neighbors) if the difference in one of their coordinates ( $\mathrm{x}$ or $\mathrm{y}$ ) is 0 while in the other, 1 . The connectivity can be defined in a recursive way: for edge point $\mathrm{p}, \mathrm{q}$, and $\mathrm{r}, \mathrm{p}$ is connected to $\mathrm{q}$ if $\mathrm{p}$ is 4-connected to $\mathrm{q}$, or $\mathrm{p}$ is 4-connected to $\mathrm{r}$ and $\mathrm{r}$ is connected to $\mathrm{q}$. 8connectivity and m-connectivity are similarly defined (Gonzalez and Woods, 1992). For simplicity, we use 4connectivity in this paper. We also assume that thinning operation has been done on the edge map so that all the edges are one pixel wide.

To illustrate the algorithm, let's first consider the simple case of an 8 by 8 edge map with all the edge pixels connected (Fig. 1: shaded pixels are edge pixels). The algorithm will do a first raster scan on the edge map and fill in water at the first edge pixel encountered that has less than 2 neighbors, i.e., start at an end point. In Fig.1 the pixel with label " 1 " is the first end point encountered. (In case all edge pixels have at least two neighbors, i.e., no end points but all loops, e.g., Fig. 3, then start at the first unfilled edge pixel during the second scan. So it is necessary to have two raster scans to avoid possible miss). The waterfront then flows along the edges in the order indicated by the numbers. Note that when there are more 
than one possible paths to go at one point, the waterfront will fork (at pixel "6" and "9" in Fig. 1).

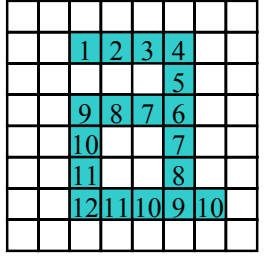

Fig. 1

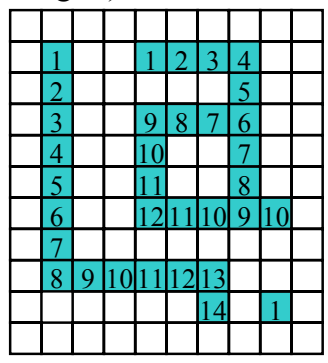

Fig. 2
One can see that this algorithm can be regarded as a simulation of "flooding of connected canal systems (i.e., connected edges)", hence the name "water-filling algorithm". (This shall not be confused with the term "water-filling paradigm" in information theory, which is about channel allocation of signal energy by noise energy distribution. See Cover and Thomas, 1991). The assumptions implied by the algorithm include: i) we have unlimited water supply at the starting pixel; ii) water flows at a constant speed in all directions; iii) water front will stop only at a dead-end, or at a point where all possible directions have been filled.

When there are more than one set of connected edges in the edge map (e.g., the case in Fig.2), the algorithm will fill all the sets independently either in sequential or in parallel.

As water fills the canals (edges), various information are extracted, which are stored as the feature primitives. Feature vectors can then be constructed based on these feature primitives. The time complexity of this algorithm is linear, proportional to the number of edge points in the image (Also see Zhou et al., 1999).

\section{Feature Extraction}

\subsection{Feature primitives}

Feature primitives are the quantities associated with or calculated from an image that can serve as bases for constructing feature vectors, often through using their statistics or entropies. Feature primitives can be used as feature vector directly as well, but often they are not compact enough. For example, $\mathrm{H}$ and $\mathrm{S}$ values (in HSV color space) are the feature primitives for the feature: color moments or color histogram; co-occurrence matrices are the feature primitives for the co-occurrence texture features, most of which are moments, correlations, and entropies (Haralick et al., 1973); and wavelet transform coefficients can be regarded as feature primitives for wavelet based texture features such as wavelet moments.

In our case, we propose the following quantities as primitives for edge/structural features:

\section{1). Filling time}

Filling time is the time for water to fill a set of connected edges. For Fig. 1 through 3, the filling times are $\{12\},\{14,12,1\}$, and $\{12\}$, respectively. Using different starting pixels, the filling time can vary in a range of $[\mathrm{t}, 2 \mathrm{t}]$, where $\mathrm{t}$ is the minimum filling time among all possible selection of the starting pixels. This is easily proved as follows: denote the starting pixel that gives the minimum filling time $\mathrm{t}$ as $\mathrm{S}$. Since we assume water runs in all possible directions with a constant speed, water can reach $\mathrm{S}$ from any other starting pixel in time $\mathrm{t}$, and then the waterfront can go from $\mathrm{S}$ to reach all other unfilled pixels within time $t$. So the filling time from any other starting pixels is less or equal to $2 \mathrm{t}$.

To minimize the variation in filling time due to selection of starting pixels, we can impose additional constraints on the selection of starting pixels (e.g., choose only the end points), or choose different starting pixels and average the results. To achieve scaling invariance, normalize the filling time according to the image size. For example, divide filling time by (width + height).

\section{2). Fork count}

Fork count is the total number of branches the waterfront has forked during the filling of a set of edges. If we consider the initial waterfront as one branch to start with, then the fork count for Fig. 1 through 3 are $\{3\},\{1$, $3,1\}$, and $\{6\}$, respectively. If we choose an end pixel as starting pixel whenever possible, fork count is invariant to starting pixel selection, hence invariant to rotation. Also if multiple waterfronts collide at one intersection, even though the water does not actually fork, the potential forks should be counted to achieve the invariance. E.g., an extra fork is counted both at the upper " 9 " and the lower "10" in Fig. 3; but none at " 12 ', since it is not a potential fork point in any way).

\section{3). Loop count}

Loop count is the number of simple loops (or, "simple cycles" as defined in Corman et al., 1997, p. 88) in a set of connected edges. For example, in Fig. 1 through 3, the loop counts are $\{1\},\{0,1,0\}$, and $\{3\}$, respectively. Loop count is invariant to rotation.

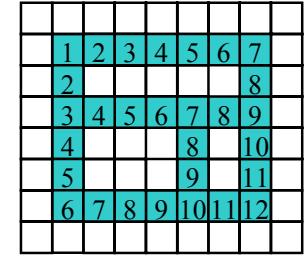

Fig. 3

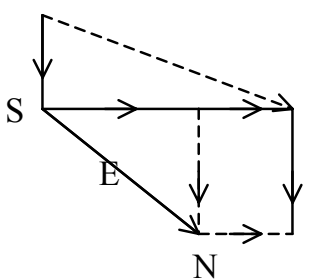

Fig. 4
To get the loop count during the water-filling process, we make use of the following "Theorem of Splashes": 
If we assume that when two waterfronts collide, we see one "splash"; or more generally, when $n$ waterfronts collide at one time, we see n-1 "splashes" (think it as n-1 waterfronts collide with the first waterfront sequentially).

Then the number of splashes $=$ the number of simple loops.

For example, in Fig. 3, three splashes are recorded at time "9", "10", and "12". Hence the loop count is 3 .

Proof: Treat the set of connected edges as a graph; and regard the starting point, the fork points, and the collision/splash points as the nodes of the graph; and the water branches as the edges. For example, the corresponding graph for Fig. 3 is shown in Fig. 4. Then the water-filling process is a traverse of the graph, and the total number of splashes is the total number of revisits to some of the nodes, which is the number of simple cycles/loops in the graph. Or more rigorously:

Wherever there is a splash, remove one of the two edges that lead to the splash. (For more than one splash at one node, treat one splash at a time). This will not affect the filling process in the sense that water can still reach all the nodes, since we only eliminated the redundant water supply paths (dashed edges in Fig. 4 are the ones removed). Finally we will obtain a spanning tree of the graph rooted at the starting pixel (solid edges in Fig. 4). The reason that this is a spanning tree is because: every node is reachable with one path from the root since we eliminated the redundant water supply paths; and, if at time $\mathrm{T}$, an edge $\mathrm{E}$ is the only edge to reach node $\mathrm{N}$ (Fig. 4), this edge $E$ will never be removed later on to make node $\mathrm{N}$ unreachable, since at the time when water hits node $\mathrm{N}$, all the paths leaving node $\mathrm{S}$ should have already been filled, so no further splash could occur at node $\mathrm{S}$ to remove edge E. Finally, From graph theory, if we add back the removed edges one at a time to a tree, we will get one simple loop for each edge inserted. Therefore number of splashes $=$ number of removed edges $=$ number of simple loops.

The above theorem provides a way of recording loop counts within the water-filling process with very little overhead computation.

\section{4). Water amount}

Water amount is the total amount of water used to fill up the set of edges in terms of number of pixels. So it is the edge pixel count. In Fig. 1 through 3, the water amounts are $\{18\},\{14,18,1\}$, and $\{29\}$, respectively.

\section{5). Horizontal (vertical) cover}

Horizontal (vertical) cover is the width (height) of the rectangular bounding box of the set of edges. In Fig. 1 through 3 , the horizontal covers are $\{5\},\{6,5,1\}$, and $\{7\}$, respectively.

\section{6). Longest horizontal (vertical) flow}

Longest horizontal (vertical) flow is the longest horizontal (vertical) edge in the set of connected edges. For Fig. 1 through 3, the longest vertical flows are $\{6\}$, $\{8,6,1\}$, and $\{6\}$, respectively.

Note that there exist many other possibilities on selecting the feature primitives. But the final selection should depend upon the specific application, i.e., what information is important and most discriminative toward the classification.

\subsection{Edge/structural features}

Based on the feature primitives in 4.1, we can then construct edge/structural features from their statistics. For example: moments (e.g., average filling time); order statistics (e.g., maximum loop count); distributions (e.g., water amount histogram); etc. In the following we discuss some examples with the emphasis on their meanings from a human perception point of view.

\section{1). (MFT\&FC) MaxFillingTime and the associated ForkCount}

MaxFillingTime is defined as $\max \{$ filling times\}. For Fig. 1 through 3, the MaxFillingTime is 12, 14, and 12, respectively. And the associated ForkCount is 3, 1, and 6

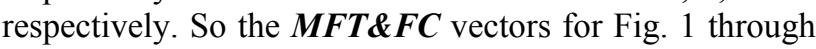
3 are $(12,3),(14,1)$, and $(12,6)$, respectively.

MFT\&FC are features most probably associated with a salient object in the image. The $\boldsymbol{M F T}$ conveys a rough measure of the size (edge length) of this object, while the associated ForkCount gives measure of complexity of the structure of the object (complexity of the edges).

\section{2). (MFC\&FT) MaxForkCount and the associated FillingTime}

Similarly defined as $\boldsymbol{M F T \& F C}$, these are also features most probably associated with a salient object in the image. The MFT conveys a rough measure of the complexity of the object. This object may or may NOT be the same object as the previous one. For Fig. 1 and 3, the $\boldsymbol{M F C \& F T}$ is the same as the MFT\&FC. But for Fig. 2,

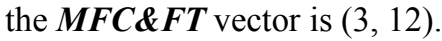

\section{3). (FTH\& FC) FillingTime Histogram and the associated averaged ForkCount within each bin}

This is a global feature on all sets of connected edges in the edge map. It represents the edge map by the distribution of edge "length". Noise or changing background with short edges may only affect part of the histogram, leaving the portion depicting the salient objects unchanged. Thus by proper weighting of the components (e.g. by relevance feedback), we could achieve robust retrieval. 


\section{4). (FCH\& FT) ForkCount Histogram and the associated averaged FillingTime within each bin}

This is also a global feature with multiple components. It represents the edge map by the distribution in edge complexity.

\section{5). (GLC\&MLC) GlobalLoopCount and MaxLoopCount}

GlobalLoopCount is defined as sum \{loop counts\}. MaxLoopCount is max $\{$ loop counts\}. This feature vector can capture structural information such as the windows in the build images. Or can be used toward character detect and recognition applications.

Note again that there can be many other possible ways to construct feature vectors, such as the moments of filling times, fork counts, loop counts, or water amounts, etc.

Regarding scaling invariance, even though we could do normalization with regard to the image size for some of features to achieve scaling invariance, the edge detector itself may not be scaling invariant. For example, at a small scale with low resolution, the edge detector can fail to extract the details in the structure where it could at a higher resolution. So if retrieval among images with different sizes is desired, then an important issue is to select or develop consistent edge detector across different resolutions.

\section{Experiments and Evaluations}

We have tested our new features on six data sets, a total of more than 20,000 images. One set is from COREL library. Four sets are from MPEG-7 testing data sets. Our experiments focus on real world images and "retrieval by example" scenarios. The edge maps are obtained by Sobel filtering followed by a thinning operation (Jain, 1989, p. 382). By using Sobel filters, we want to make the point that even with the simplest edge detector, the water-filling features can still give good results. Water-filling features used in these experiments are $\boldsymbol{M F T \& F C}$ (see Section 4

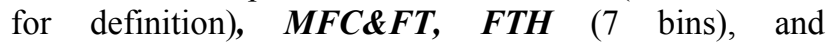
GLC $\boldsymbol{M} \boldsymbol{M L C}$ - a total of 18 feature components per image. Our system uses a two-level weighted Euclidean distance for image matching, with the weights dynamically adjusted by relevance feedback from the user. For details, see Rui, et al., 1998 and 2000.

\subsection{City/Building images and Landscapes}

The first experiment is carried out on a set of 92 images from Mpeg-7 test set (Fig 5). Fig 6 and 7 show the retrieval results using as a query a city/building image and a landscape image, respectively.

To evaluate the performance, comparison between texture features, namely wavelet moments, and water- filling features is performed for city/building image retrieval. First, 17 out of the 92 images are labeled as "buildings" by a human subject. Each of them is then used as a query image in the retrieval process using wavelet or water-filling features. The number of correct hits out of the top 10 and top 20 returns is counted into two scatter plots in Fig 8, where each point in the plot corresponds to one of the 17 queries, and its coordinates are the number of hits by using texture and water-filling features, respectively. One can see that most of the points are above the diagonal, indicating that water-filling features outperform texture features (wavelet moments) in most cases. In top 10 returns, water-filling features give an average of 6.2 correct hits, versus 4.7 by wavelet moments. In top 20 returns, the figures are 9.0 versus 7.1.
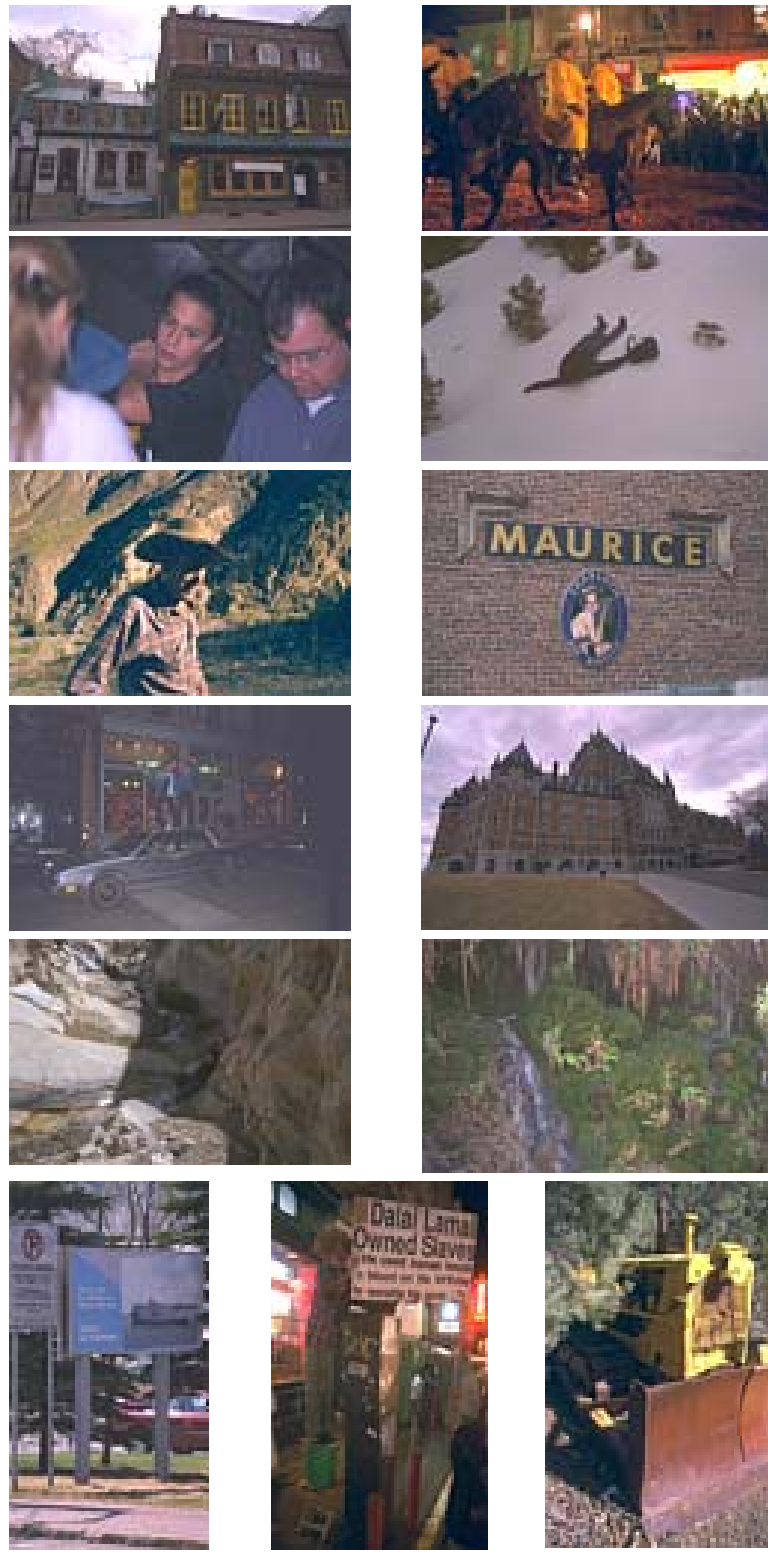

Fig. 5 Random picks from a set of 92 images. 

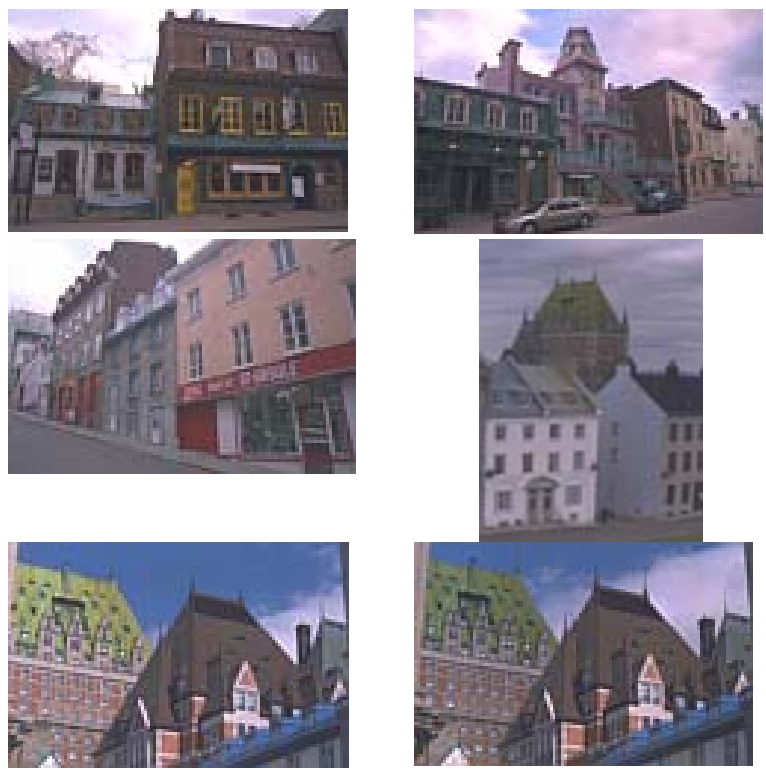

Fig. 6 Top 5 retrieved based on water-filling features. (The top-left image is the query.)
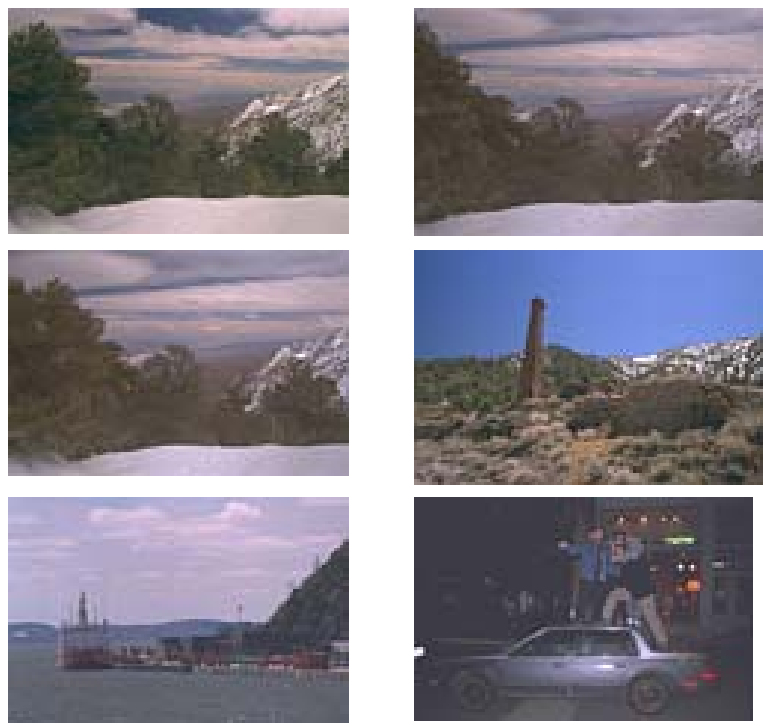

Fig. 7 Top 5 retrieved based on water-filling features.

(The top-left image is the query.)
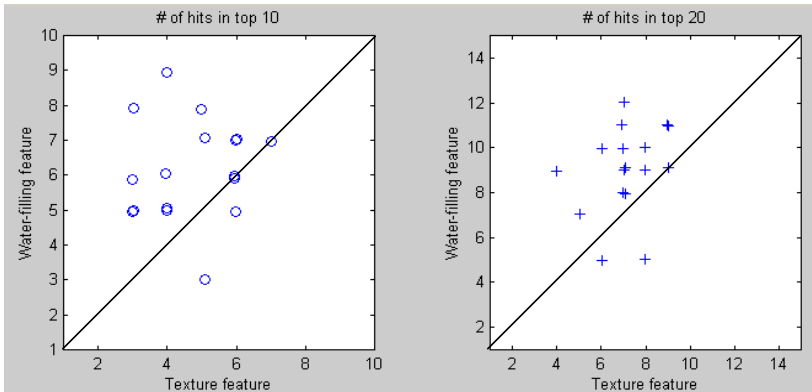

Fig. 8 Compare Texture features (wavelet moments) and water-filling features
To evaluate the performance, comparison between texture features, namely wavelet moments (Smith and Chang, 1995), and water-filling features is performed for city/building image retrieval. First, 17 out of the 92 images are labeled as "buildings" by a human subject. Each of them is then used as a query image in the retrieval process using wavelet or water-filling features. The number of correct hits out of the top 10 and top 20 returns is counted into two scatter plots in Fig 8, where each point in the plot corresponds to one of the 17 queries, and its coordinates are the number of hits by using texture and water-filling features, respectively. One can see that most points are above the diagonal, indicating that water-filling features outperform texture features (wavelet moments) in most cases. In top 10 returns, water-filling features give an average of 6.2 correct hits, versus 4.7 by wavelet moments. In top 20 returns, the figures are 9.0 versus 7.1 .

Intuitively, windows, doors, and the skylines expose the characteristics of City/building images, which can be captured by the proposed water-filling features.
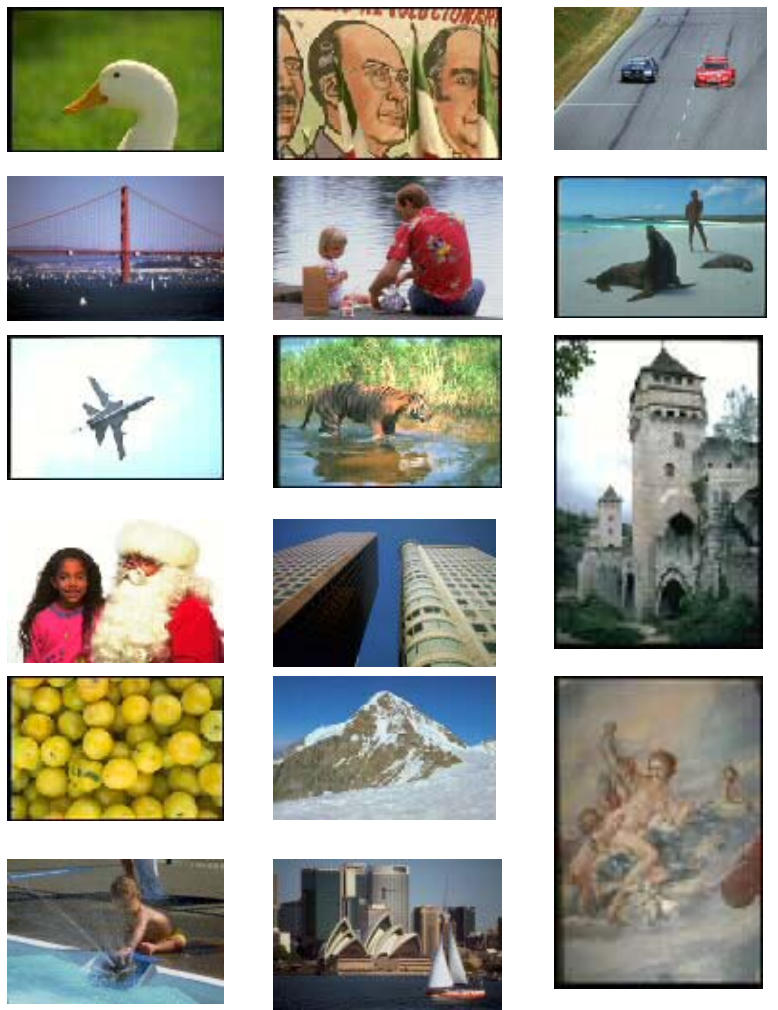

Fig. 9 Random picks from a set of 17,695 images

\subsection{Images with clear structure: Birds and Airplanes in the sky}

The second retrieval task is performed on the COREL data set of 17,695 images (Fig. 9). Query images are those with clear edge structures (Fig. 10). One can see that retrieval by shape would not be helpful in such cases because from different angles the objects appear 
dramatically different in shape. The water-filling features can be effective in dealing with these cases by capturing the rough size, internal structural complexity, and global edge distribution, etc. But since the information represented in the water-filling features is rough, it can still mistake birds for airplanes, and vice versa, in some cases (e.g., Fig. 11). For a more systematic performance comparison, see Table 1 below, which shows the comparison in terms of averaged number of hits in top 10 , 20, 40 and 80 returns for 100 airplanes and 100 eagles as the query images, respectively. Note that although the averaged numbers are comparable between the two features, the underlying matching mechanisms are very different: here texture features mainly depend upon the dominant uniform background for matching, while waterfilling features look at the edge patterns. Fig. 11 reveals the fact that the returns by water-filling features and texture features are totally different, which indicates that water-filling features capture new information that are not captured by texture features; which also explains why adding water-filling can enhance the overall performance (See Section 5.3 for the experimental results).
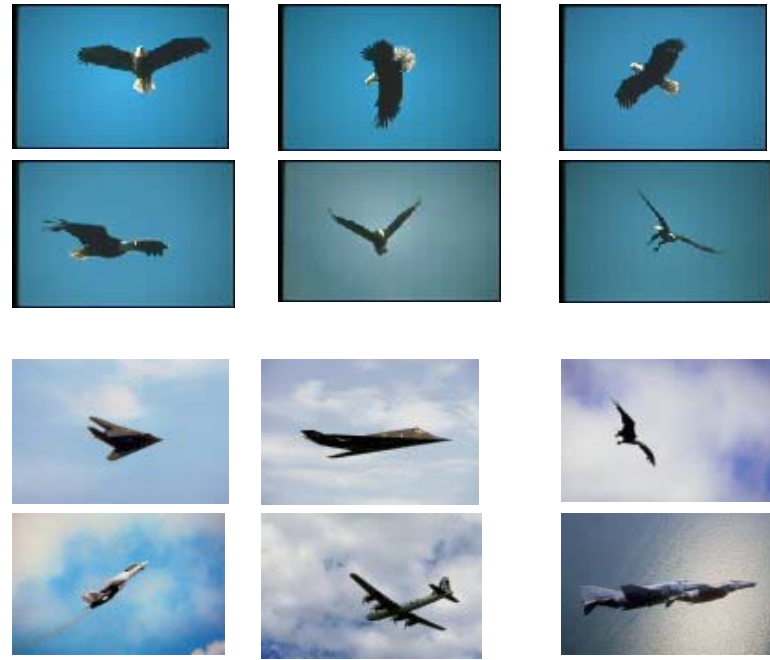

Fig. 10 "Eagles" and "Airplanes":

Top 5 retrieved based on water-filling features.

(The top-left is the query image.)

Table 1. Water-filling (WF) versus Wavelet Variances (WV) (100 airplanes and 100 eagles as query images)

\begin{tabular}{|c|c|c|c|c|}
\hline Airplanes & \#Hit in top 10 & Top 20 & Top 40 & Top 80 \\
\hline WF & 3.56 & 6.29 & 10.92 & 18.03 \\
\hline WV & 3.32 & 5.75 & 9.94 & 17.07 \\
\hline Eagles & \#Hit in top 10 & Top 20 & Top 40 & Top 80 \\
\hline WF & 2.65 & 3.33 & 4.91 & 6.79 \\
\hline WV & 1.98 & 2.82 & 4.43 & 6.58 \\
\hline
\end{tabular}

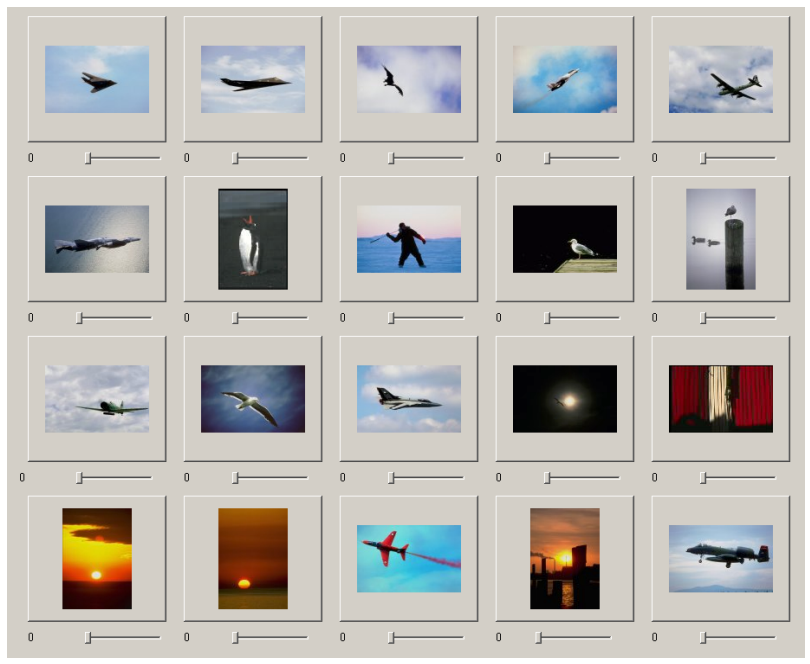

(a)

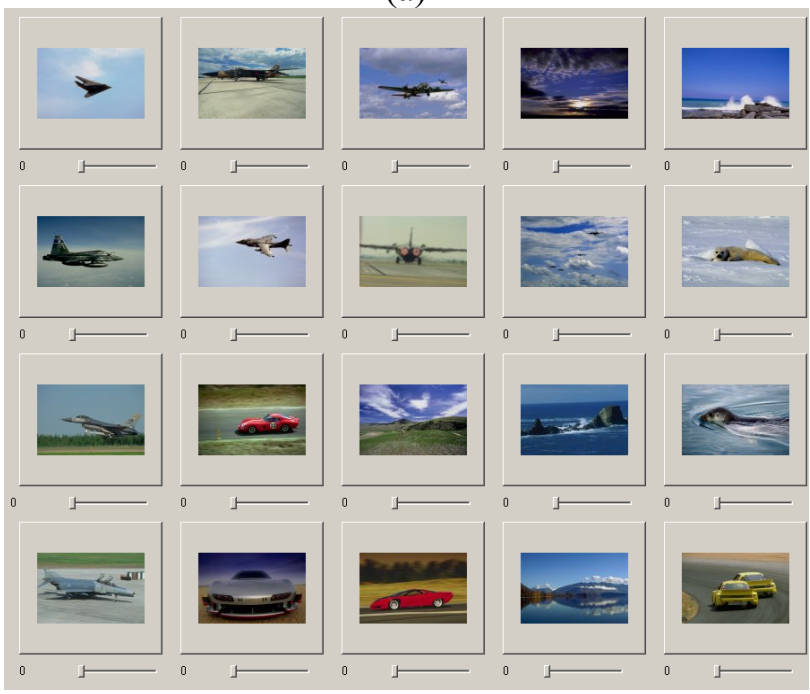

(b)

Fig. 11 Retrieving airplanes by (a) water-filling features; (b) texture features (The top-left is the query image.)

\subsection{High-level concepts: Horses, Tigers and Cars}

For high-level concepts such as horses, tigers, or cars, water-filling features alone performs very poorly, (so does texture features); since they do not contain enough information, or the right information, to characterize the salient object - a good example is "tigers in the bushes"Table 2 shows averaged performance using 100 tiger images as queries. The number of hits in top 80 is less than 4 (a random sampler will on average retrieval about $100 \times 80 / 17695+1=1.45$ in top 80 , including the query).

The new features are also tested under the relevance feedback environment. Our two-level dynamic feature weighting scheme via relevance feedback can be briefly described as follows: two levels of weights - low level for the feature vector component, high level for different features (e.g., color feature, texture feature, and structural 
feature, see Fig. 12)—are adjusted during each retrieval based on the user's feedback regarding whether or not and how much a returned image is relevant to what he/she is looking for. The resulting weighting matrix conveys how important different features/components are to the current retrieval task. Usually the optimal weighting matrix varies from query to query, and this agrees with our intuition: if a user considers cars of varies colors as relevant, maybe weights on color features should not be the highest; whereas if all relevant images are red cars, color can receive the highest weights. (For details on algorithms and performance analysis, see Rui, et al., 1998 and 2000).

To test the impact of introducing water-filling features into the system, 20 randomly selected "horse" images are used as initial queries, and retrieval and relevance feedback are performed based on feature sets \{waterfilling\}; \{color; texture\}; and \{color; texture; waterfilling\}, respectively. Table 3 shows the averaged numbers of hits in top 20 after: 1 . the initial query (when all weights are set to be equal); 2 . one round of relevance feedback; 3. several rounds - usually 3 8 - of feedback until convergence. The results indicate that even though water-filling features alone perform very poorly, after combined with other features, it can improve the overall performance, especially after dynamic weighting of the features through relevance feedback technique.

Table 2. Water-filling (WF) versus Wavelet Variances (WV) (100 tigers as query images)

\begin{tabular}{|c|c|c|c|c|}
\hline Tigers & \#Hit in top 10 & Top 20 & Top 40 & Top 80 \\
\hline WF & 1.42 & 1.85 & 2.41 & 2.98 \\
\hline WV & 1.38 & 1.76 & 2.27 & 3.21 \\
\hline
\end{tabular}

Table 3. Feature performance with relevance feedback (C: Color; T: Texture; S: Water-filling)

\begin{tabular}{|c|c|c|c|}
\hline Horses & $\{\mathrm{S}\}$ & $\{\mathrm{C} ; \mathrm{T}\}$ & $\{\mathrm{C} ; \mathrm{T} ; \mathrm{S}\}$ \\
\hline Initial & 2.25 & 7.05 & 7.45 \\
\hline $1 \mathrm{RF}$ & 3.45 & 10.10 & 11.20 \\
\hline Converge & 3.85 & 12.95 & 15.35 \\
\hline
\end{tabular}

Fig. 12 (a) shows the converged result after 5 rounds of relevance feedback using color and texture features. Twolevel of weights are shown in the right part of the figure. While adding water-filling features, the converged results are in (b), note that not only more horses are retrieved, but also some white horses are also retrieved. This is possibly due to the reason that water-filling features are capable of pulling the system out of the convergence where all horses are confined by certain color by adding horses of different color but the same edge structures into the top 20 returns. (c) and (d) are the same kind of comparison but for "tiger" images, which are less structured in term of edges. This time water-filling features did not contribute significantly but still brought in two more hits in top 20 returns.

It is discovered that in many cases color contribute the most during the retrieval. Indeed for the examples in Fig. 12 , color plays the most important role. However this is

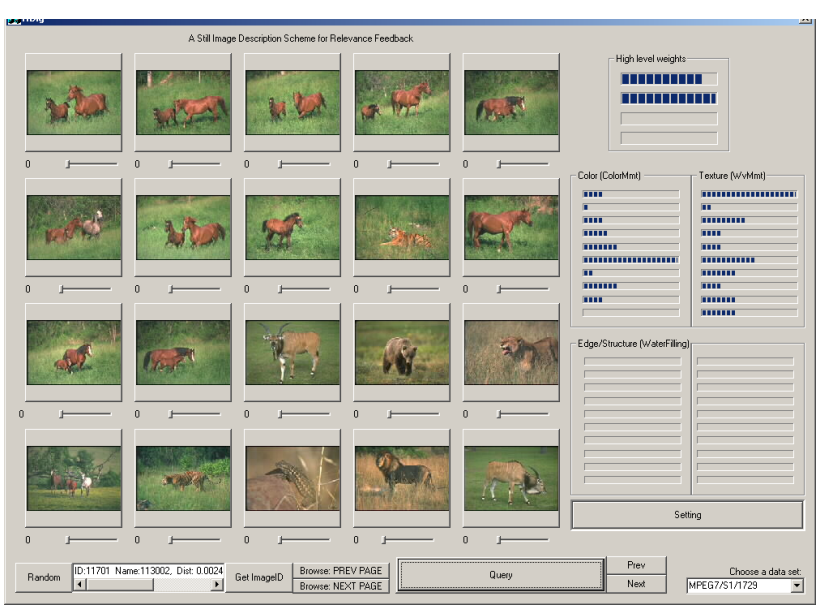

(a)

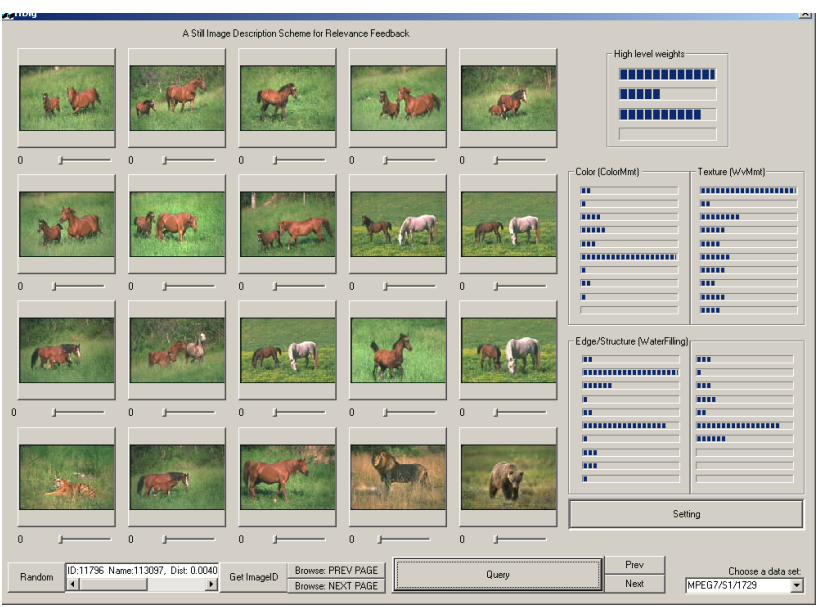

(b)

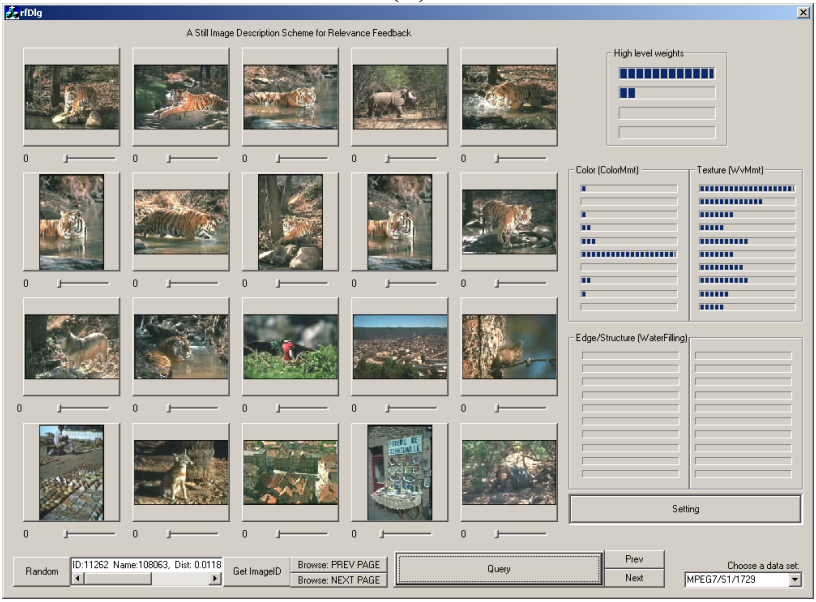

(c) 


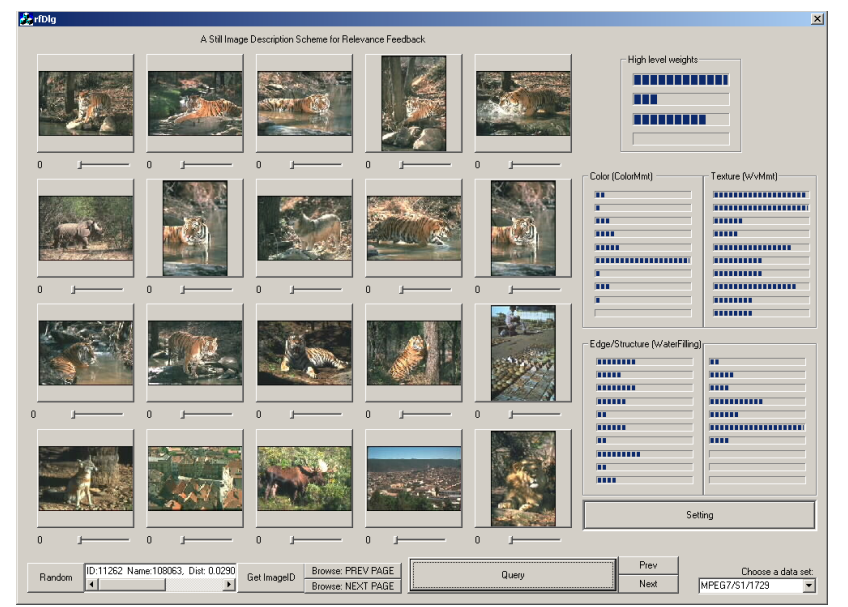

(d)

Fig. 12 Feature performance comparisons after relevance feedback. (a) and (c) are for color and texture features only; (b) and (d) are for color, texture and water-filling features. Showing to the right are the two-level feature weights calculated based on user relevance feedback.

not always the case. The relative importance of different features actually depends upon the user's interest. In the next experiment, the user is assumed to look for "racing car" images. Fig. 13 (a) through (c) show the results using \{color\}; \{color; texture\}; and \{color; texture; waterfilling\}, respectively. Note the increase in the number of hits as well as the change in the relative weights on different features. Color clearly is not an important criterion for the user in this case. This fact is captured by the system through a lower weight on color features (In Fig. 13(c), the three bars on the top right correspond to color, texture and water-filling, respectively).

One issue worth pointing out is that for example, in the case of retrieving horse images, we are not actually getting "horses" from the system, but rather something like "objects of red and/or white color, of certain size, and

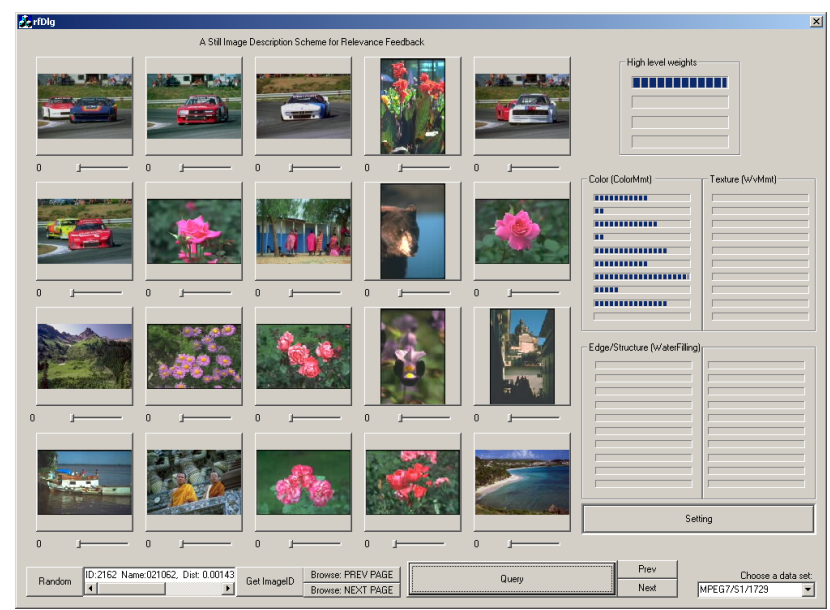

(a)

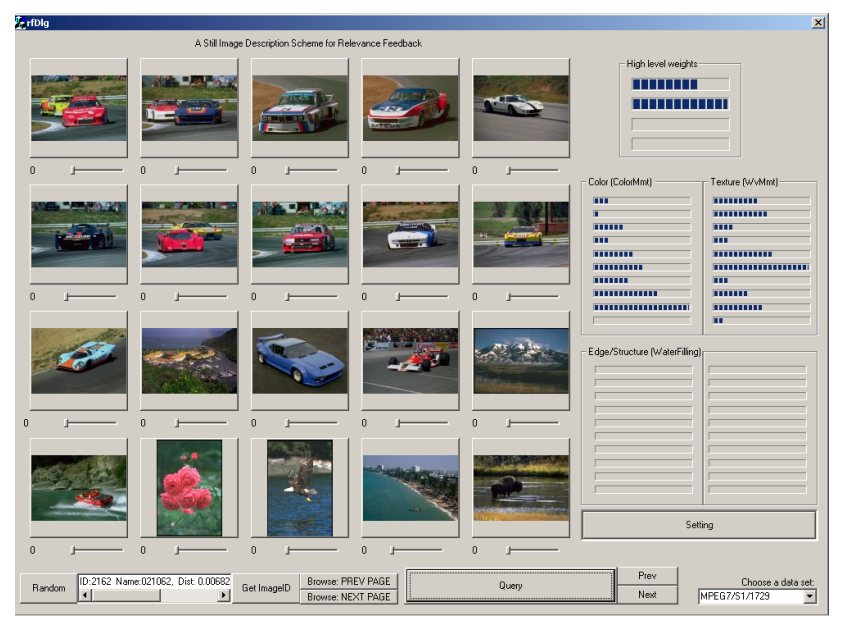

(b)

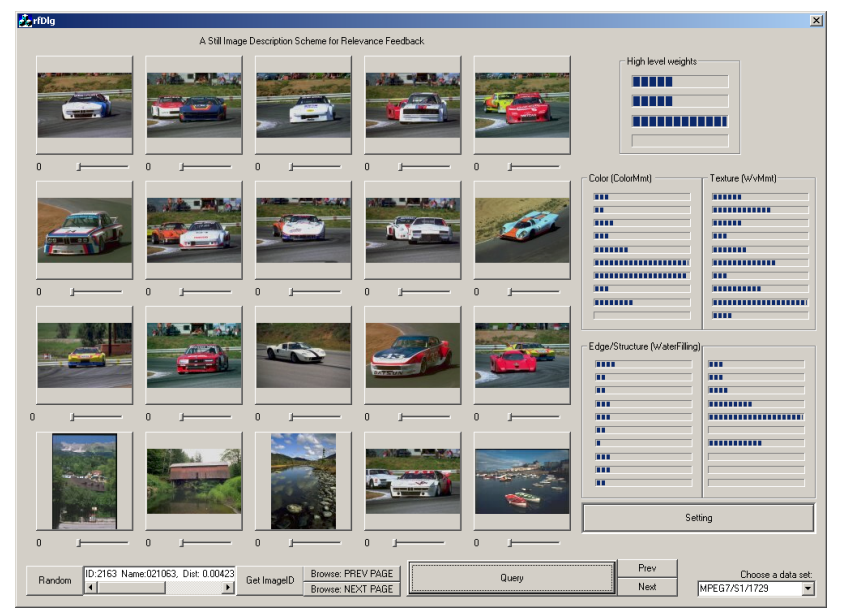

(c)

Fig. 13 Performance comparisons after relevance feedback. (a) is for color moments only; (b) is for color and texture features; (c) is for color, texture and waterfilling features.

of certain edge structure, in a green background of certain texture..." which happens to be horse images in this specific database. Some of the features may not be relevant to the concept in the user's mind, such as the green background in this case for the retrieval of "horses". This is a typical problem with global features and it is an important issue to investigate in our system as well as any other CBIR systems. An obvious direction to go is to do segmentation and local feature extraction. But this is out of the scope of this paper.

\section{Conclusions}

In this paper we proposed structure features for CBIR systems, and applied the water-filling algorithm to extract edge/structure features directly from edge maps. Based on the experimental results, the new features possess the following characteristics: (1) they can represent edge/structural information embedded in the edge maps, 
such as edge length (rough measure), edge connectivity and complexity, loop structure, etc., which are proven to be effective in CBIR, even with simple Sobel filters as the edge detector. (2) They are translation, rotation, and scaling-invariant to certain extent (see Section 4 for detailed analysis). (3) They can catch some information that matches human perception in structure, which may not be carried by texture or shape features. (4) The proposed edge/structural features are more general than texture or shape features in that they require neither uniform texture region nor a closed shape contour. They can be effective on non-uniform real-world images and natural scenes. (5) Since water-filling features are extracted from the binary edge map, edge detection algorithm plays an important role in these features. Also since edge extraction process is a lossy transformation in terms of information contents, false positive during retrieval is sometimes rather high, which indicates the integration with other features is necessary for large databases. Future work includes exploring edge detectors that are robust under noisy condition and different resolutions; and detailed analysis of the water-filling features for different classes of images.

Acknowledgement: This work was supported in part by National Science Foundation Grant CDA 96-24396 and EIA 99-75019.

\section{Reference}

Corman, T. H., C. E. Leiserson, R. L. Rivest, 1997. Introduction to algorithms, McGraw-Hill, New York.

Cover, T. and J. Thomas, 1991, Elements of Information Theory, John Wiley \& Sons, Inc., Toronto.

Flickner, M. et al., 1995, Query by image and video content: The qbic system, IEEE Computers.
Gonzalez, R. C. and Woods, 1992, Digital Image Processing, Addison-Wesley

Haralick, R. M., K. Shanmugam, and I. Dinstein, 1973, Texture feature for image classification, IEEE Trans. SMC, Nov.

$\mathrm{Hu}, \mathrm{M} . \mathrm{K}$., 1962, Visual pattern recognition by moment invariants", IRE Trans. Information Theory, 8

Iqbal, Q. and J. K. Aggarwal, 1999, Applying perceptual grouping to content-based image retrieval: Building images, Proc. IEEE CVPR'99, 42-48

Jain, A. K., 1989, Fundamentals of Digital Image Processing, Prentice Hall

Laine, A., J. Fan, 1993. Texture classification by wavelet packet signatures. IEEE Trans. Pattern Anal. Machine Intell. 15, 1186-1191

Persoon, E. and K. S. Fu, 1977, Shape discrimination using Fourier descriptors, IEEE Trans. SMC, Mar.

Ratan, A. L., et. al., 1999, A framework for learning query concepts in image classification, Proc. IEEE CVPR'99, 423429

Rui, Y., T. S. Huang, M. Ortega, and S. Mehrotra, 1998, Relevance Feedback: A Power Tool in Interactive ContentBased Image Retrieval", IEEE Tran on Circuits and Systems for Video Technology, Vol 8, No. 5, Sept., 644-655

Rui, Y., T. S. Huang, 2000, Optimizing Learning in Image Retrieval, Proc. IEEE CVPR'2000.

Smith, J. R. and Chang, 1995, Transform features for texture classification and discrimination in large image databases, Proc. IEEE ICIP'95

Vailaya, A., A. K. Jain and H. J. Zhang, 1998, On image classification: City Images vs. Landscapes, Pattern Recognition, vol. 31, December, 1921-1936

Zahn, C. T. and Roskies, 1972, Fourier descriptors for plane closed curves, IEEE Trans. Computers

Zhou, X. S., Y. Rui, and T. S. Huang, 1999, Water-filling: a novel way for image structural feature extraction, Proc. ICIP99 